\title{
High Accuracy Attitude Control System Design for Satellite with Flexible Appendages
}

\author{
Wenya Zhou, ${ }^{1,2}$ Haixu Wang, ${ }^{1}$ Zhengwei Ruan, ${ }^{1}$ Zhigang Wu, ${ }^{1,2}$ and Enmei Wang ${ }^{1}$ \\ ${ }^{1}$ School of Aeronautics and Astronautics, Dalian University of Technology, Dalian 116023, China \\ ${ }^{2}$ State Key Laboratory of Structural Analysis for Industrial Equipment, Dalian University of Technology, Dalian 116023, China \\ Correspondence should be addressed to Wenya Zhou; zwy@dlut.edu.cn
}

Received 27 August 2014; Revised 29 September 2014; Accepted 29 September 2014; Published 4 November 2014

Academic Editor: Hui Zhang

Copyright (c) 2014 Wenya Zhou et al. This is an open access article distributed under the Creative Commons Attribution License, which permits unrestricted use, distribution, and reproduction in any medium, provided the original work is properly cited.

\begin{abstract}
In order to realize the high accuracy attitude control of satellite with flexible appendages, attitude control system consisting of the controller and structural filter was designed. When the low order vibration frequency of flexible appendages is approximating the bandwidth of attitude control system, the vibration signal will enter the control system through measurement device to bring impact on the accuracy or even the stability. In order to reduce the impact of vibration of appendages on the attitude control system, the structural filter is designed in terms of rejecting the vibration of flexible appendages. Considering the potential problem of in-orbit frequency variation of the flexible appendages, the design method for the adaptive notch filter is proposed based on the in-orbit identification technology. Finally, the simulation results are given to demonstrate the feasibility and effectiveness of the proposed design techniques.
\end{abstract}

\section{Introduction}

Since the satellite was designed with compact structure and simple configuration at early stage, satisfied attitude control accuracy can be achieved while considering the satellite as the rigid body during the design of attitude control system [1-3]. However, the satellite structure is becoming more complicated and the appendage is becoming bigger in size and more in quantity in order to satisfy the modern space missions. These flexible appendages are apt to vibrate due to the attitude maneuver of satellite or external disturbance. When the vibration signal enters the attitude control system of satellite through the measurement device, the attitude control accuracy will be influenced or even the instability of system might be caused $[4,5]$. Many scholars have performed deeper and further studies with respect to the design of high accuracy attitude control system of satellites with flexible appendages [6-8].

During the design of satellite structure, the vibration modes of its flexible appendages are usually obtained through ground test. While performing the design of attitude control system for satellite, frequency isolation method, that is, the bandwidth of attitude control system will be kept far away from the first order mode vibration frequency of flexible appendages, will be adopted in order to avoid the influence of appendages vibration on the attitude control [9]. But when the flexible appendage is big, the first order mode vibration frequency will be close to the instinct frequency of the rigid; therefore, the above method will not be applicable any more. Aiming at such low frequency vibration rejection problem, structural filter is one of the effective methods to reject the vibration [10, 11]. In [12-14], Wie et al. presented the design method of structural filter. However, these methods are developed for fixed frequency. When the satellite is operating in orbit, the vibration frequency of flexible appendages might change due to the failure or damage. Such variation of frequency is hardly predicable and will directly threaten the control accuracy of satellite attitude. Therefore it is powerless to use the above structural filter to improve the control accuracy.

In this paper, with the pitch channel as an example, the attitude control system including the adaptive structural filter is developed for the satellite with flexible appendages. In order to reduce the impact of vibration of appendages on 
the attitude control system, the structural filter is designed to reject the vibration of flexible appendages. Considering the potential problem of in-orbit frequency variation of the flexible appendages, the design method for the adaptive notch filter is proposed based on the in-orbit identification technology. The in-orbit vibration frequency of appendages can be obtained by above technology and structural filter can be generated adaptively so that the purpose of improving the control accuracy of satellite attitude can be realized. This is the main contribution of this paper. The simulation results show that the proposed attitude control system can still achieve good attitude control accuracy when the vibration frequency of the flexible appendages changed.

\section{Attitude Control System Design}

2.1. Pitch Channel Dynamics Model for Satellite. The equation of pitch channel dynamic model for satellite with flexible appendages is given by

$$
\begin{gathered}
I_{y} \ddot{\theta}-2 \sum_{i=1}^{4} B_{r i} \ddot{q}_{i}(t)=T_{y}, \\
\ddot{q}_{i}(t)+2 \zeta \omega_{i} \dot{q}_{i}(t)+\omega_{i}^{2} q_{i}(t)-B_{r i} \ddot{\theta}=0,
\end{gathered}
$$

where $\theta$ is the pitch-axis pointing error output, $I_{y}=$ $7750 \mathrm{~kg} \cdot \mathrm{m}^{2}$ is the satellite pitch inertia, $T_{y}$ is the pitchaxis reaction wheel control torque input, $q_{i}$ is the $i$ th order displacement in broad sense for antenna flexible vibration, $\omega_{i}$ is the $i$ th flexible mode frequency in $\mathrm{rad} / \mathrm{s}$, and $\zeta$ is the passive damping ratio assumed to be 0.01 . $B_{r i}$ is the $i$ th coupling coefficient; it describes the influence degree of the $i$ th vibration mode and the center of the antenna rigid. Equation (1) can be expressed by Laplace transform:

$$
\begin{gathered}
I_{y} s^{2} \theta(s)-2 B_{r 1} s^{2} q_{1}(s)-\cdots-2 B_{r n} s^{2} q_{n}(s)=T_{y}(s), \\
q_{i}(s)=\frac{B_{r i} s^{2}}{s^{2}+2 \zeta \omega_{i}+\omega_{i}^{2}} \theta(s), \quad(i=1,2, \ldots, n),
\end{gathered}
$$

where $s$ is the Laplace transform variable.

Substituting (3) into (2), simplify the equation and select the fore 4 order modes; the transfer function of pitch channel is obtained as follows:

$$
\frac{\theta(s)}{T_{y}(s)}=\frac{1}{I_{y} s^{2}-2 \sum_{i=1}^{4}\left(B_{r i}^{2} s^{4} /\left(s^{2}+2 \zeta \omega_{i} s+\omega_{i}^{2}\right)\right)} .
$$

Data of ground test for the fore 4 order vibration mode of flexible appendages are summarized in Table 1.

2.2. Control Law Design. The system block diagram of pitch channel control system for satellite is shown as in Figure 1.

Since the external disturbance $d$ is in cycle, there is no static error in the system output under normal situation. PD control law is used:

$$
u=K_{P} e+K_{D} \dot{e}
$$

TABLE 1: Modal data for the pitch axis.

\begin{tabular}{lcc}
\hline Mode number & $\omega_{i} \mathrm{rad} / \mathrm{s}$ & $B_{r i}$ \\
\hline 1 & 0.569 & 61.09 \\
2 & 4.438 & -3.18 \\
3 & 12.475 & 0.806 \\
4 & 23.281 & -0.459 \\
\hline
\end{tabular}

PD controller can be designed by the root locus method, where $K_{P}, K_{D}$ are the proportion and differential coefficients, respectively, with $K_{P}=68, K_{D}=1000$.

Without the structure filter, the open loop magnitude curve of pitch channel is shown in Figure 2 with solid line.

It can be seen that when the frequency is higher than $1.77 \mathrm{rad} / \mathrm{s}$, the system magnitude will go above the $0 \mathrm{~dB}$ line again. Obviously, the vibration signal, whose frequency is higher than $1.77 \mathrm{rad} / \mathrm{s}$, will be amplified once it enters the control system. It will seriously affect the accuracy of the attitude control. Therefore, we need to design structure filter to reduce the effects on the pitch attitude control channel caused by the vibration.

2.3. Structure Filter Design. Notch filter is one of the common structural filters. It has a good inhibition for fixed frequency vibrations and there is no effect on the system bandwidth. Therefore, the notch filter is used in this study for rejecting the vibration of appendages and its general expression is as follows:

$$
\frac{s^{2} / \omega_{z}^{2}+2 \zeta_{z} / \omega_{z}+1}{s^{2} / \omega_{p}^{2}+2 \zeta_{p} / \omega_{p}+1}
$$

where $\omega_{z}, \omega_{p}, \zeta_{z}, \zeta_{p}$ are the undetermined parameters of filter. Notch filter for different purpose can be obtained through adjusting these parameters such as band elimination filter, band-pass filter, low-pass filter, high-pass filter, and phase leading/lagging filter. In order to reduce the influence from the fore two order modes on attitude control system, the expressions of notch filter are selected based on the frequency corresponding to the peak:

$$
\begin{aligned}
& S_{1}(s)=\frac{s^{2} / \omega_{z_{1}}^{2}+2 \zeta_{z_{1}} / \omega_{z_{1}}+1}{s^{2} / \omega_{p_{1}}^{2}+2 \zeta_{p_{1}} / \omega_{p_{1}}+1}, \\
& S_{2}(s)=\frac{s^{2} / \omega_{z_{2}}^{2}+2 \zeta_{z_{2}} / \omega_{z_{2}}+1}{s^{2} / \omega_{p_{2}}^{2}+2 \zeta_{p_{2}} / \omega_{p_{2}}+1},
\end{aligned}
$$

where $\zeta_{z_{1}}=\zeta_{z_{2}}=0.01, \zeta_{p_{1}}=\zeta_{p_{2}}=0.99, \omega_{z_{1}}=2.88, \omega_{p_{1}}=$ $(1-\sigma) \omega_{z_{1}}, \omega_{z_{2}}=4.71$, and $\omega_{p_{2}}=(1-\sigma) \omega_{z_{2}}$.

The above filters are called minimum-phase notch filters. $\sigma$ is the frequency regulating factor and equals 0.25 . The magnitude plot of open loop system with notch filters is shown in Figure 2 with dashed line. Obviously, the magnitude which is higher than the bandwidth frequency is suppressed below the $0 \mathrm{~dB}$ line through introducing the notch filter.

Select $\theta=0.5^{\circ}$ as the input of system and there is a signal in the following format in the measurement loop. Figure 3 


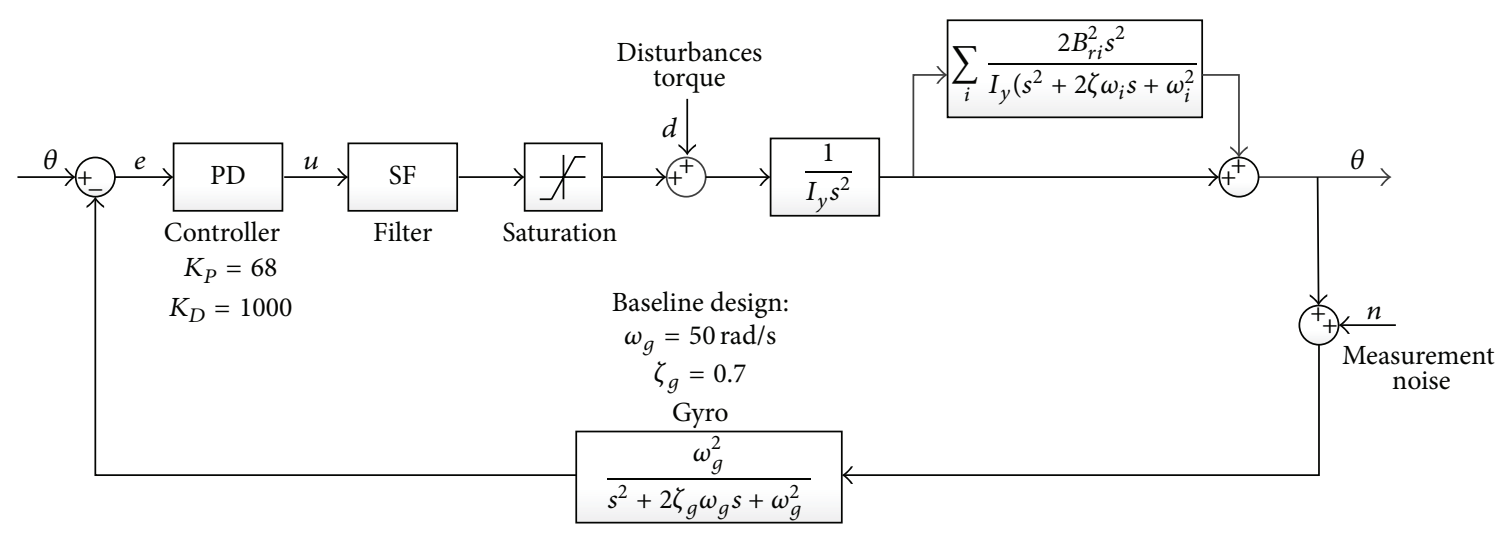

FIGURE 1: Block diagram of the model pitch-axis control system.

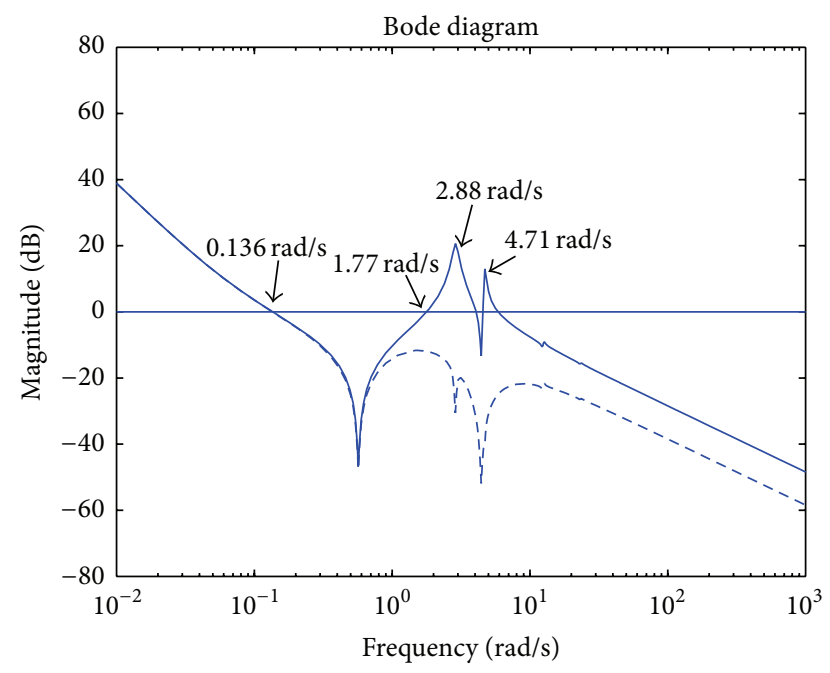

FIGURE 2: Bode magnitude plot of open loop system with/without the notch filter.

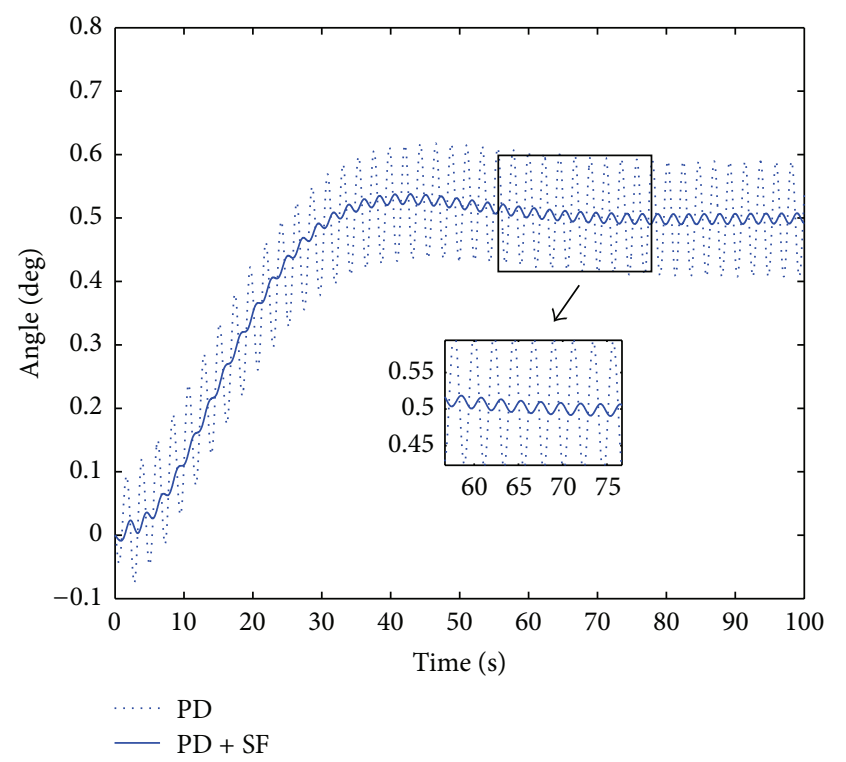

FIGURE 3: Response curves of pitch angle.

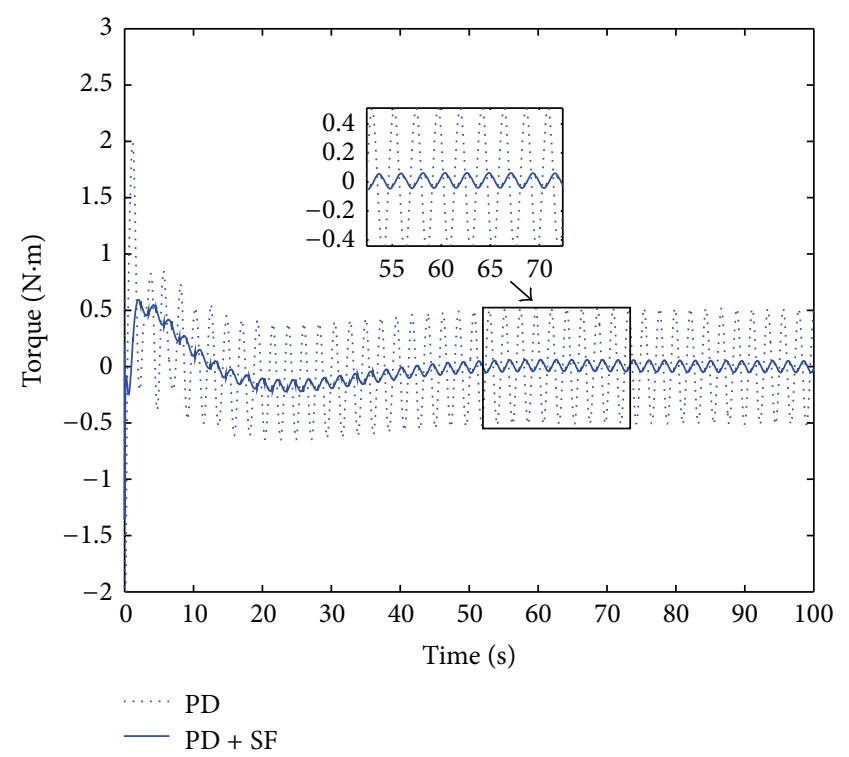

FIGURE 4: Control torque curves.

gives the system output response curves. The dashed line denotes the system output with only PD control and the solid line with PD control plus notch filter. The corresponding torque curves are shown in Figure 4. The meanings of dashed line and solid line are the same as the above mentioned.

And the measurement noise is $n=A_{1} \sin \left(\omega_{d} t\right)$, where $A_{1}=0.1$ and $\omega_{d}=2.80 \mathrm{rad} / \mathrm{s}$.

From Figures 3 and 4, the output responds and control torque are improved evidently by introducing the notch filter.

\section{Adaptive Notch Filter Design}

The flexible appendages are susceptible to be damaged because the satellite will work in the environment of vacuum, weight loss, temperature variation, and intense radiation so that the vibration mode might be changed. If this happens, the system response curve is given as in Figure 5 by assuming that the first order vibration frequency $\omega_{1}$ is changed from $0.569 \mathrm{rad} / \mathrm{s}$ to $0.435 \mathrm{rad} / \mathrm{s}$. 


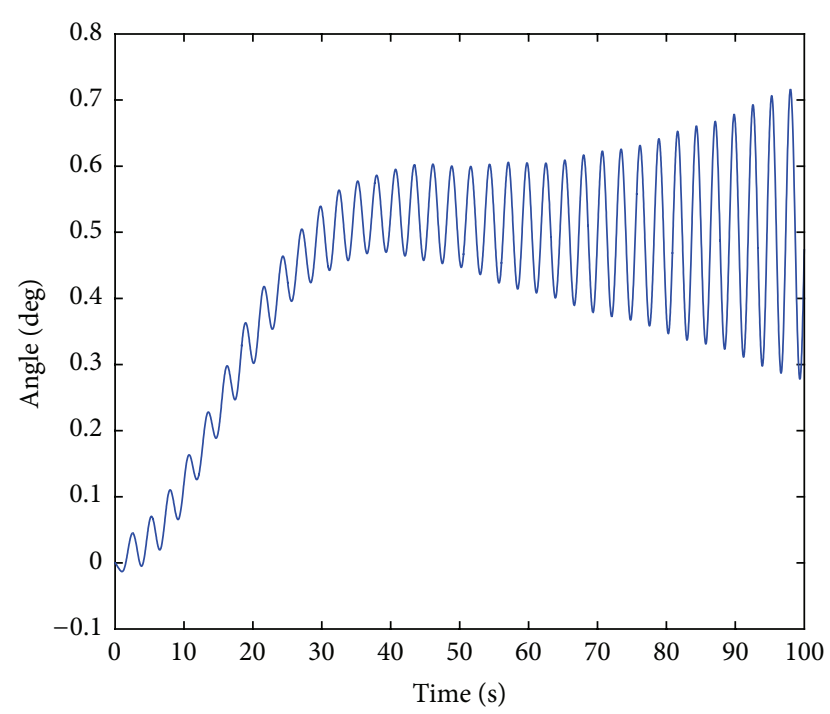

FIGURE 5: System response curve when $\omega_{1}=0.435 \mathrm{rad} / \mathrm{s}$.

It can be seen that when the first order vibration frequency of flexible appendages changes, the pitch angle response curve is divergent. This shows that the notch filter is effective for certain fixed frequency signal; however, when this frequency changes, the filter will be powerless. In order to ensure that the high accuracy of attitude control still can be achieved under the above condition, it is required to estimate the vibration frequency of in-orbit flexible appendages and to redesign the structural filter based on the estimated value.

3.1. LSM Identification. The least squares method (LSM) is often used to model identification [15]. Assume the difference equation as

$$
A\left(z^{-1}\right) y(k)=B\left(z^{-1}\right) u\left(k-T_{d}\right)+\varepsilon(k),
$$

where $A\left(z^{-1}\right), B\left(z^{-1}\right)$ are discrete unit operator polynomials and $\varepsilon(k)$ is external disturbance. Consider

$$
\begin{aligned}
& A\left(z^{-1}\right)=1+a_{1} z^{-1}+a_{2} z^{-2}+\cdots+a_{n_{a}} z^{-n_{a}}, \\
& B\left(z^{-1}\right)=b_{0}+b_{1} z^{-1}+b_{2} z^{-2}+\cdots+b_{n_{b}} z^{-n_{b}},
\end{aligned}
$$

where $n_{a}, n_{b}$ are the orders of structure, respectively, and $T_{d}$ the order of pure delay link.

The identification process is to determine the $n_{a}+n_{b}+$ $T_{d}$ parameters including $a_{1}, a_{2}, \ldots, a_{n_{a}} ; b_{0}, b_{1}, \ldots, b_{n_{b}}$ through detected inputs and outputs.

Rewrite the identification model equation (8) into

$$
\begin{aligned}
y(k)= & -a_{1} y(k-1)-a_{n_{a}} y\left(k-n_{a}\right) \\
& +b_{0} u\left(k-T_{d}\right)+b_{n_{b}} u\left(k-T_{d}-n_{b}\right)+\varepsilon(k) \\
= & \varphi^{T}(k) \phi+\varepsilon(k),
\end{aligned}
$$

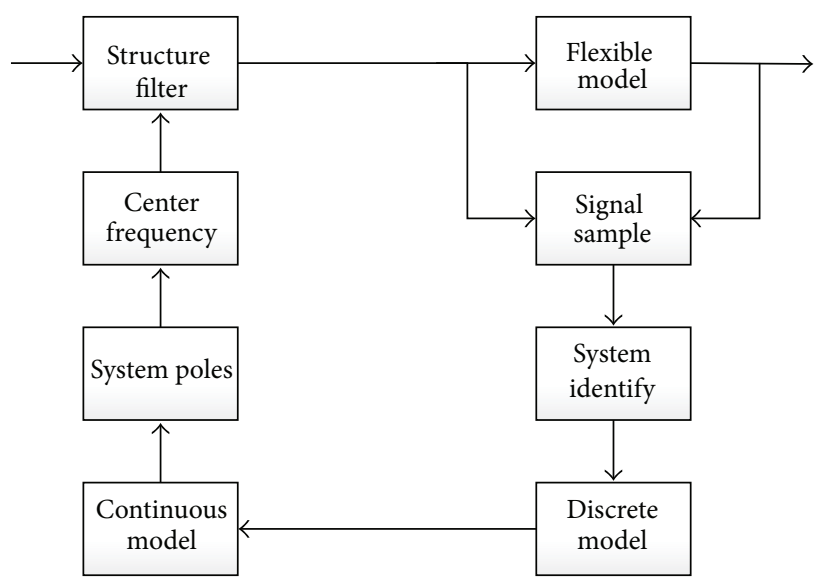

FIGURE 6: Realization process of adaptive notch filter.

where $\varphi(k)$ is the observation vector composed of input and output signals and $\phi$ is the coefficient matrix composed of $a_{1}, a_{2}, \ldots, a_{n_{a}} ; b_{0}, b_{1}, \ldots, b_{n_{b}}$. Consider

$$
\begin{gathered}
\varphi(k)=\left[-y(k-1), \ldots,-y\left(k-n_{n_{a}}\right),\right. \\
\left.u\left(k-T_{d}\right), \ldots, u\left(k-T_{d}-n_{n_{a}}\right)\right]^{T}, \\
\phi=\left[a_{1}, \ldots, a_{n_{a}}, b_{0}, \ldots, b_{n_{b}}\right]^{T} .
\end{gathered}
$$

The coefficients of object $a_{1}, a_{2}, \ldots, a_{n_{a}} ; b_{0}, b_{1}, \ldots, b_{n_{b}}$ can be obtained based on measured input and output signals in $n$ groups; that is, $\widehat{\phi}=\left(\Phi^{T} \Phi\right)^{-1} \Phi^{T} Y$, where $Y=$ $\left[\begin{array}{llll}y(1) & y(2) & \cdots & y(n)\end{array}\right]^{T}, \Phi=\left[\begin{array}{llll}\varphi^{T}(1) & \varphi^{T}(2) & \cdots & \varphi^{T}(n)\end{array}\right]^{T}$.

3.2. Design of Adaptive Structural Filter. The vibration model can be obtained by the above LSM identification. The center frequency $\omega_{n}$ of adaptive filter can be calculated through solving poles of vibration model. Consider

$$
\omega_{n}=\frac{1}{T_{s}} \sqrt{\ln z_{r} \ln z_{r}^{*}},
$$

where the sampling time $T_{s}=0.1, z_{r}, z_{r}^{*}$ is one pair of conjugate poles with the maximum imaginary part. The center frequency of adaptive filter is $\omega_{n}=2.27 \mathrm{rad} / \mathrm{s}$. It can be known that the system magnitude is slightly increasing with the vibration frequency of flexible appendages moving forward. This makes it more difficult to design the notch filter. The minimum-phase notch filter was designed by similar way and the frequency regulating factor is taken as $\sigma=0.4$. The expression of filter is given as follows:

$$
S_{1}(s)=\frac{s^{2} / \omega_{n}^{2}+2 \zeta_{1} / \omega_{n}+1}{s^{2} /(1-\sigma)^{2} \omega_{n}^{2}+2 \zeta_{2} /(1-\sigma) \omega_{n}+1},
$$

where $\zeta_{1}=0.01$ and $\zeta_{2}=0.99$.

The realization process of adaptive notch filter is shown in Figure 6 [16]. 


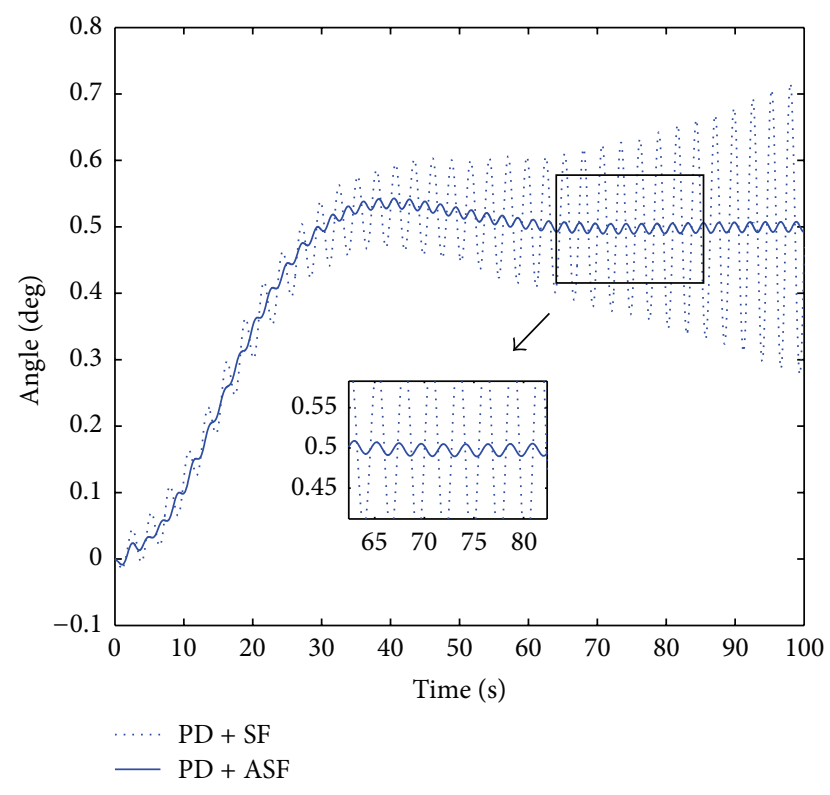

FIGURE 7: Response curves of pitch angle.

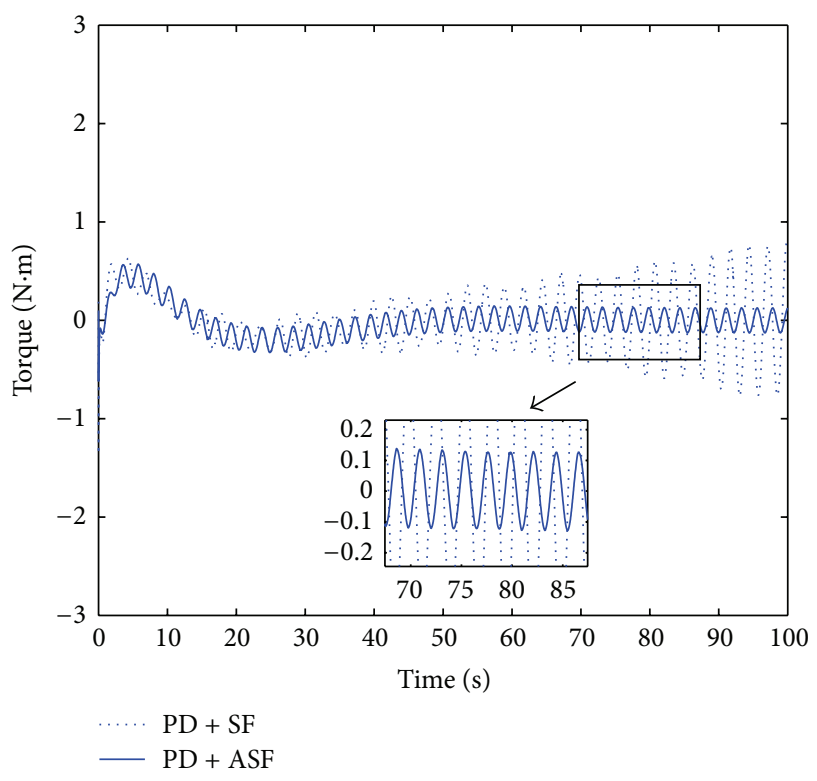

FIGURE 8: Control torque curves.

When the in-orbit frequency of flexible appendages varies, the output response of attitude control system with adaptive notch filter is shown in Figure 7. The control torque curve is shown in Figure 8.

In Figure 7, solid line and dashed line represent the system output curves with notch filter and that with adaptive notch filter, respectively. It is obvious that the system output with notch filter is divergent, whereas that with adaptive notch filter is convergent and control accuracy can be maintained. In Figure 8, the meanings of dashed line and solid line are the same as the above mentioned. The control torque can be maintained stable with the adaptive notch filter.
The amplitude of torque curve is increasing while comparing with that in Figure 4. This is caused by the increased gain amplitude of the system due to the changes of flexible vibration frequency.

\section{Conclusions}

Notch filter is introduced into the control system design to improve the attitude control accuracy in this study in which the pitch angle attitude control system for satellite with flexible appendages is taken as the object. It shows that notch filter can well reject the appendages vibration with fixed frequency, but it will become powerless when the vibration frequency of flexible appendages varied. During the design of notch filter, the identification technology is adopted to obtain the real-time estimate of vibration frequency of flexible appendages and the notch filter will be generated adaptively based on the estimate value. This kind of filter can well reject the potential uncertain flexible vibration so that the high accuracy attitude control can be ensured and system reliability is improved.

\section{Conflict of Interests}

The authors declare that there is no conflict of interests regarding the publication of this paper.

\section{References}

[1] C. D. Jilla and D. W. Miller, "Satellite design: past, present and future," International Journal of Small Satellite Engineering, 1997.

[2] J. R. Wertz, Spacecraft Attitude Determination and Control, vol. 73, D. Reidel Publishing Company, 1978.

[3] B. Bonnard, "Control of the attitude of a rigid satellite," Rairo Automatique Systems Analysis and Control, vol. 16, no. 1, pp. 8593, 1982.

[4] S. P. Tan, Y. J. Lei, and L. Tang, “The influence of system noises on the attitude control stability of flexible satellite," Aerospace Control and Application, vol. 36, no. 1, pp. 42-45, 2010.

[5] H. Zhang, X. Zhang, and J. Wang, "Robust gain-scheduling energy-to-peak control of vehicle lateral dynamics stabilisation," Vehicle System Dynamics, vol. 52, no. 3, pp. 309-340, 2014.

[6] M. Azadi, S. A. Fazelzadeh, M. Eghtesad, and E. Azadi, "Vibration suppression and adaptive-robust control of a smart flexible satellite with three axes maneuvering," Acta Astronautica, vol. 69, no. 5-6, pp. 307-322, 2011.

[7] E. Findlay, J. R. Forbes, H. H. T. Liu et al., Investigation of Active Vibration Suppression of a Flexible Satellite Using Magnetic Attitude Control, University of Toronto Press, 2011.

[8] Q. Hu and G. Ma, "Variable structure control and active vibration suppression of flexible spacecraft during attitude maneuver," Aerospace Science and Technology, vol. 9, no. 4, pp. 307-317, 2005.

[9] X. L. Chen, D. Yang, and K. Zhai, "A flexible satellite attitude control system design," Journal of Harbin Institute of Technology, vol. 40, no. 5, pp. 1-5, 2008.

[10] H. Zhang, Y. Shi, and J. Wang, "On energy-to-peak filtering for nonuniformly sampled nonlinear systems: a markovian jump system approach," IEEE Transactions on Fuzzy Systems, vol. 22, no. 1, pp. 212-222, 2014. 
[11] S. Funke, K. Mehlhorn, and S. Näher, "Structural filtering: a paradigm for efficient and exact geometric programs," Computational Geometry: Theory and Applications, vol. 31, no. 3, pp. 179-194, 2005.

[12] B. Wie, Q. Liu, and F. Bauer, "Classical and robust $H_{\infty}$ control redesign for the hubble space telescope," Journal of Guidance, Control, and Dynamics, vol. 16, no. 6, pp. 1069-1077, 1993.

[13] B. Wie and K.-W. Byun, "New generalized structural filtering concept for active vibration control synthesis," Journal of Guidance, Control, and Dynamics, vol. 12, no. 2, pp. 147-154, 1989.

[14] B. Wie, J. A. Lehner, and C. T. Plescia, "Roll/yaw control of a flexible spacecraft using skewed bias momentum wheels," Journal of Guidance, Control, and Dynamics, vol. 8, no. 4, pp. 447-453, 1985.

[15] S. Chen, S. A. Billings, and W. Luo, "Orthogonal least squares methods and their application to nonlinear system identification," International Journal of Control, vol. 50, no. 5, pp. 18731896, 1989.

[16] L. F. Chu, Z. G. Wu, and C. Yang, "Design and simulation of missile adaptive filter mechanism," Journal of Aeronautics, vol. 32, no. 2, pp. 195-201, 2011. 


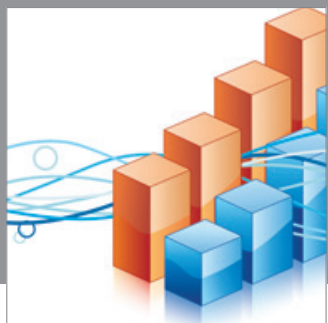

Advances in

Operations Research

mansans

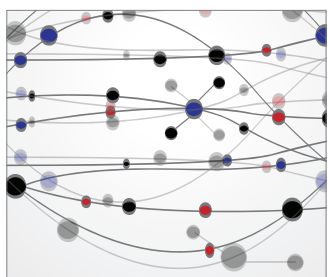

The Scientific World Journal
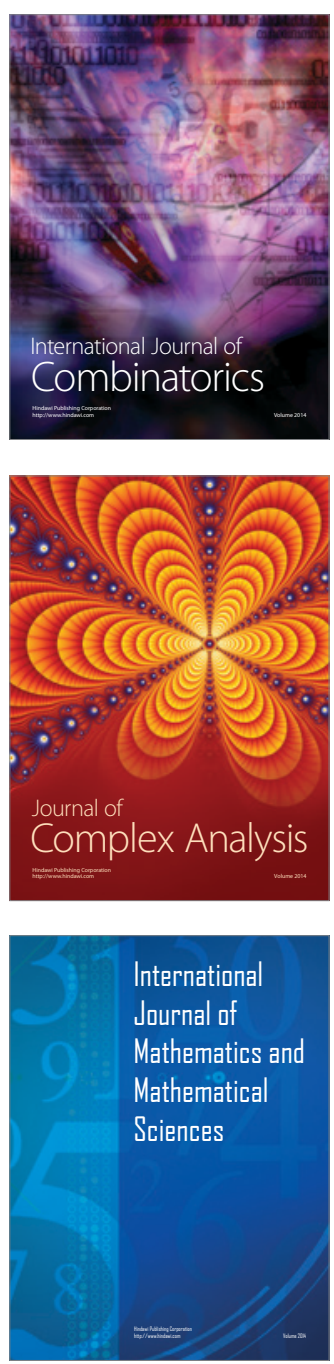
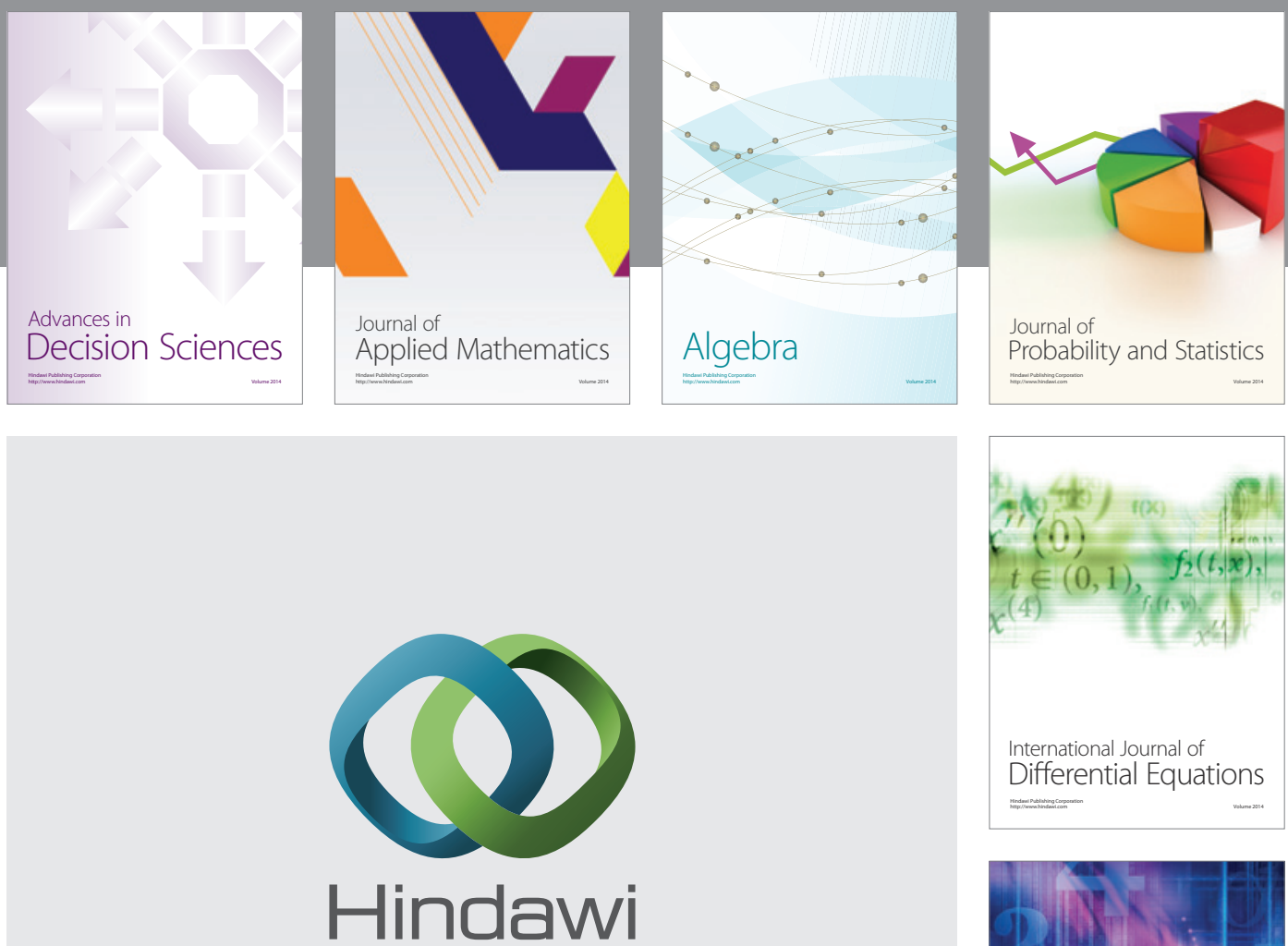

Submit your manuscripts at http://www.hindawi.com
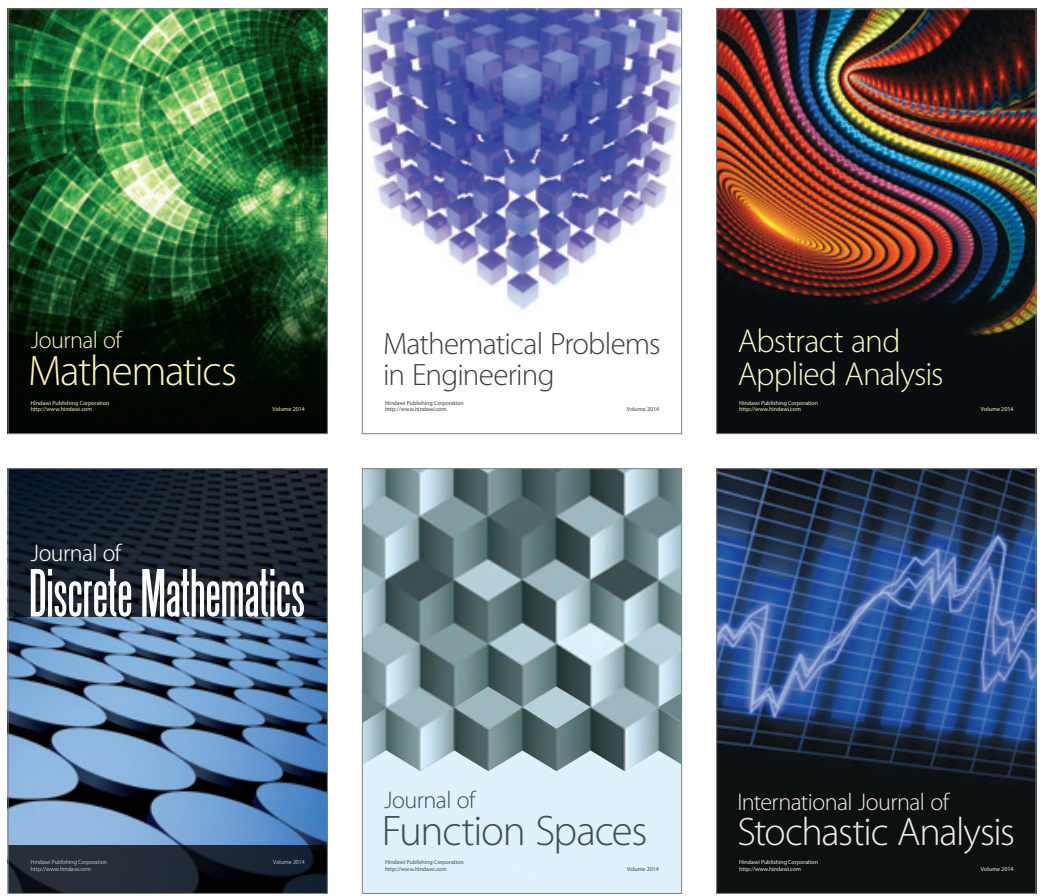

Journal of

Function Spaces

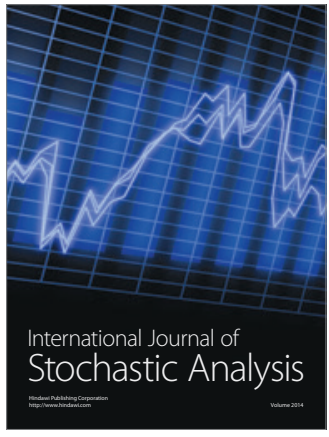

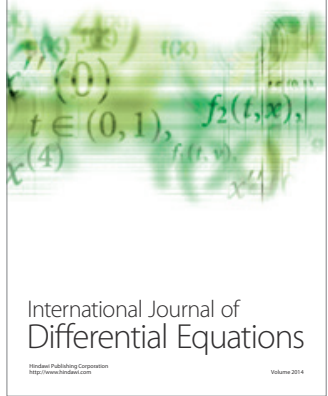
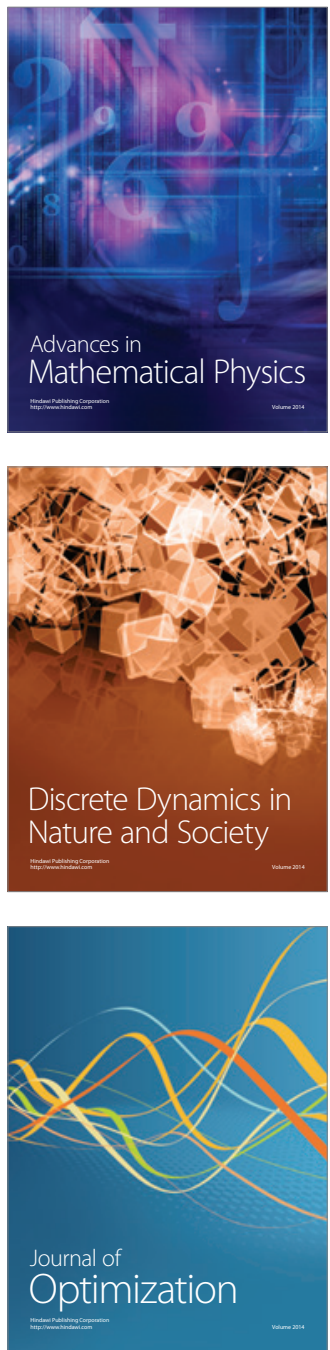